\title{
Tweaking Business Planning With Artificial Intelligence
}

\author{
Jing Rui Chen, UCSI University, Malaysia \\ P. S. Joseph Ng, UCSI University, Malaysia
}

\begin{abstract}
Griffith AI\&BD is a technology company that uses big data platform and artificial intelligence technology to produce products for schools. The company focuses on primary and secondary school education support and data analysis assistance system and campus artificial intelligence products for the compulsory education stage in the Chinese market. Through big data, machine learning, and data mining, scattered on campus and distributed systems enable anyone to sign up to join the huge data processing grid and access learning support big data analysis and matching after helping students expand their knowledge in a variety of disciplines and learning and promotion, improve the learning process based on large data sets of students, and combine AI technology to develop AI electronic devices to provide schools with the best learning experience to survive in a competitive world.
\end{abstract}

\section{KEYWORDS}

Artificial Intelligence, Artificial Intelligence Product, Big Data, Big Data System, Education System, Machine Learning, User Experience

\subsection{INTRODUCTION}

Griffith AI\&BD is a Chinese technology company dedicated to providing a high-tech new learning environment for schools and students. The company is built on a B2B strategy. Program the company's AI robot products based on the data set generated by big data. The product can analyze students' behaviour in school and help students solve problems. The product feeds data back to AI through an AI learning system, so that AI and big data complement each other, forming an ai system centred on education. The more data the AI receives, the smarter it becomes. Combining the insights of ARTIFICIAL intelligence, the speed of data analysis techniques, and access to new sources of information will lead to an unprecedented level of informed decision-making based on intelligent and accurate analysis.

If a big data company only serves students, it will not help reform the education system. Problems in the education system will still bring bad results to the growth of students. Based on integrating artificial intelligence into schools, our company integrates big data education systems into campus management. To deepen the application of artificial intelligence and data analysis in education and teaching, promote scientific diagnosis, precision teaching and personalized learning, and change the traditional education system.

Griffith AI\&BD will introduce artificial intelligence robots on campus. Combined with the campus environment, the data set based on-campus data is specially compiled, and the existing student data set of each tutoring company is loaded into the school in the form of an artificial intelligence service to form a special intelligent education system. And the learning capabilities of

\section{DOI: 10.4018/IJBSA.288541}

This article published as an Open Access article distributed under the terms of the Creative Commons Attribution License (http://creativecommons.org/licenses/by/4.0/) which permits unrestricted use, distribution, and production in any medium, provided the author of the original work and original publication source are properly credited. 
the company's AI will be used to generate new, more accurate sets of campus data. Our company will be positioned as a technology company serving schools, and its products will be various forms of artificial intelligence devices or robots as well as intelligent education systems to meet the needs of our customers. Therefore, combining educational needs with the future development of big data and artificial intelligence provides our company with a huge competitive advantage.

In addition to providing schools and customers with quality products, we also provide schools and customers with efficient use of training services. And provide after-sales maintenance and upgrade services. Each customer's analysis, data, monitoring content will be shared with the customer. And it allows customers to program and design products that are more suitable for themselves or the school environment.

Griffith AI\&BD is established in the form of a joint-stock limited company, which is composed of $20 \%$ of the founders, $20 \%$ of the partners, $10 \%$ of the technical specialists and $50 \%$ of the shareholders. Please refer to. See Section Management team for more details.

\subsection{LITERATURE REVIEW}

AI has brought wonderful transitions in the education sector, benefiting both the students and the institutions (Johnson, 2019). With the development of artificial intelligence technology, the landing of $\mathrm{AI}$ in the field of education has gradually become a trend. More and more AI enterprises or education enterprises have begun to actively explore the application scenarios of AI in the field of education. In July 2017, the Chinese government released an ambitious master plan to build the country into a powerhouse in AI innovation, especially in education. In China, related enterprises are developing a series of in-depth construction around artificial intelligence LABS, teacher training, educational robots and other basic education systems. Griffith AI\&BD targets private schools first because private schools need more innovation to generate more students and more profits.

As early as 2013, the Ministry of Education launched a new round of revision of high school curriculum standards, with more than 260 experts working for four years. The revision of the national curriculum, standards and textbooks is managed by the Ministry of Education, showing the significance of these revisions. The revised standards explicitly mention "core competencies". This educational goal is set in the context of reflecting on the relationship between ARTIFICIAL intelligence and human intelligence, human-machine collaboration, and future joint development. Chinese education has begun to realize the transformation from the traditional systematic emphasis on the integrity and structure of academic knowledge and the underplay of skill training to the improvement of students' ability and quality of thinking and the promotion of good development (Wang, 2019). More directly related to the field of AI, the revised secondary education curriculum standards include specific ai teaching content. The revised high school IT curriculum is no longer centred on computers and the Internet but focuses on data, algorithms, information systems and the information society. These all require ai products to be present in schools.

In strengthening basic science education activities, China has carried out a wide range of AI education activities, encouraged science and technology professionals across the country to participate in AI education and popularization, improved the general public's understanding of AI, and built and improved basic knowledge in all aspects. Infrastructure for ai science education. The usefulness of various artificial intelligence innovation platforms is fully reflected in popular science exhibitions and popular science museums.

AI is bringing personalized opportunities to the entire education system. Many Chinese tech companies are increasing the use of smart technology to build education systems. All kinds of education technology enterprises actively explore and make extensive use of ARTIFICIAL intelligence and other technologies, launch the construction of "smart campus" schools, develop online learning and education platforms using big data intelligence, and promote the application of ARTIFICIAL intelligence in mathematics, management and other fields. resources construction and other fields. 
Griffith AI\&BD faces and competes with competitors on two fronts. However, as an innovative company that focuses on the school market, it is easier for us to cooperate with other competitors. The competitors' analysis is shown below.

Shenzhen Tencent made its fortune in social software. Founded in 1998, it is one of the largest integrated Internet service providers in China and one of the Internet companies with the largest number of users. At present, ARTIFICIAL intelligence has been used in several Tencent products, such as WeChat speech recognition to text, listening to music, face recognition, etc., but also such as users shopping, news, behind the artificial intelligence for everyone's preferences to make relevant recommendations. Tencent also announced its AI strategy, gathering dozens of AI scientists and 70 world-class $\mathrm{AI} \mathrm{PhDs}$ from around the world to focus on research in AI fields such as machine learning, computer vision, speech recognition and natural language processing. Based on massive data of hundreds of millions of users and technical advantages in various vertical fields of the Internet, Tencent aims to build the world's top AI team.

An AI named "Jiewu", for example, is a research project between Tencent AI Lab and King of Glory and has defeated humans in a game between AI and professional players. AI Jiewu as Tencent AI Lab artificial intelligence representative work, its algorithm set several high levels of game players data, it can be continuous training, will not produce tired feeling. With the deepening research on deep reinforcement learning and multi-agent decision making, "King must realize" no longer needs to imitate human data, but through their own and their battle, further improve the micro-level and the overall view, to reach the level of the king of glory professional esports.

The technology behind Jiewu AI mainly involves two areas: supervised learning and reinforcement learning. In supervised learning, behavioural prediction functions are derived based on massive labelled training data. The advantage of this method is that it can simulate different levels of the target behaviour and achieve good anthropomorphism. The downside is an over-reliance on the data, especially the quality of the annotated data. A researcher in Tencent's AI Lab published a data report. According to the report, one day of AI enlightenment training is equivalent to 150 years of human training. Tencent already has a well-established AI technology community in China in various fields. Is one of the biggest competitors to the rest.

\section{Big Data for Education And Training}

Education and training institutions are using big data to personalize lesson plans, predict learning outcomes, and even help students find colleges and majors that fit their interests and skills. For example, Civitas' platform takes data from all parts of a university or institution to help students make more specific decisions with calculated outcomes. Its solutions also help faculty optimize their biggest tasks and more effectively engage with students.

But the education and training industry is in decline. According to media reports, China's education stocks collapsed on July 23, 2021: Popular education stocks such as New Oriental, Good Future and Highway plunged 60\%, the biggest one-day drop in history. But those shares were down $70 \%$ before the crash. Professor Larry Hsien Ping Lang, a well-known Chinese economist, said on July 24: "Education and training, the Internet, real estate, etc., will be marginalized by the market. So, in China, understand the big trends."

By 2021, at least $65 \%$ of China's top 1000 enterprises will use artificial intelligence tools such as natural language processing (NLP), machine learning (ML) and deep learning (DL) to empower $60 \%$ of areas such as customer experience, security, business use case operations management and procurement. By 2022, 80 percent of China's top 1,000 companies will invest in internal learning platforms and third-party training services, and gradually adopt new skills and changes in working styles brought by AI. With the healthy growth of China's AI market, Griffith AI\&BD expects average monthly sales to reach 500,000 yuan from the first month, with monthly sales growing by more than $3 \%$.

The world is changing rapidly due to the emergence of innovational technologies (Chae, 2019). The use of AI has increased in different enterprises. In 2019, IDC predicted that spending on AI 


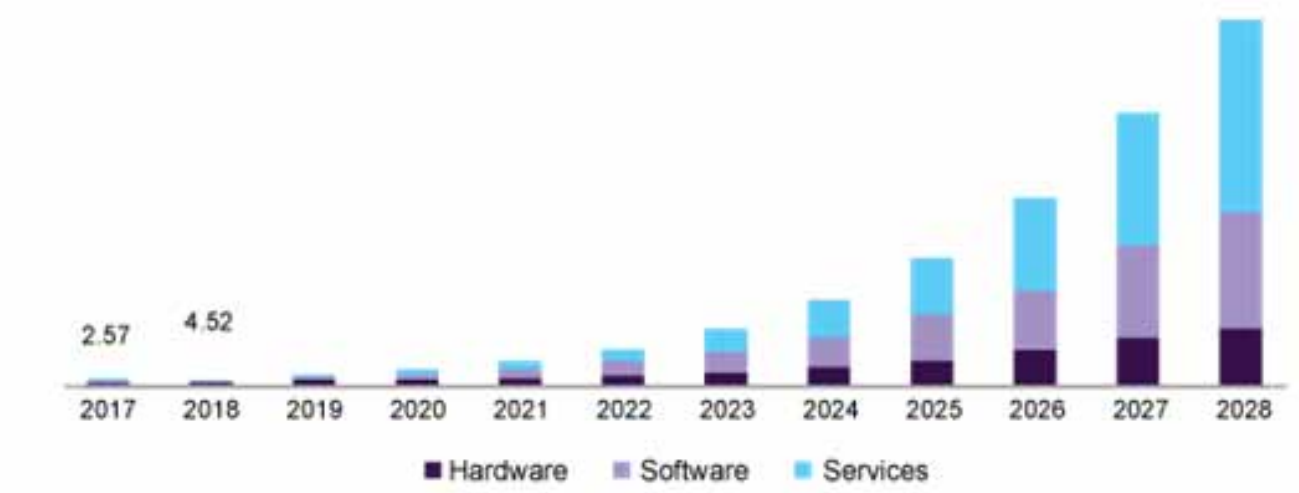

Source: waw. grindviewresearch com

technology would increase to $\$ 97.9$ billion by 2023 . The potential value of AI has only grown since the COVID-19 pandemic swept the world. At least half of organisations have adopted AI features in their organisations, according to a survey by McKinsey State of AI released in November 2020 (Bahirat, 2021).

$\mathrm{AI}$ is becoming increasingly important as organizations continue to automate daily operations and understand data sets affected by COVID-19. There are more digital connections between businesses than ever before since the lockdown and work-from-home came into force.

For these AI fields to mature, their AI algorithms need huge amounts of data. Natural language processing, for example, would not be possible without millions of human speech samples, recorded and broken down into formats that ai engines could more easily process. Big data will continue to get bigger as AI becomes a more viable option for automating more tasks -- and AI will become a bigger field as more data becomes available for learning and analysis. "AI and ML are now giving us new opportunities to use the big data that we already had, as well as unleash a whole lot of new use cases with new data types," says Glenn Gruber, senior digital strategist at Anexinet." (Casey, 2019).

And big data, as an academic term that is generally defined more broadly than AI, means large amounts of structured, semi-structured, or unstructured data, as well as a way to collect/generate, process, and analyze these data sets using non-traditional methods such as the Al approach. This data is the fuel for artificial intelligence and intelligent machines, the "new oil" (Agrawal, 2018). As a result, big data and AI are often intertwined and work together to drive the digital transformation of current society.

At present, people use a lot of technical equipment. All the time, a lot of data is being generated through these devices. To cater for this massive data, current technologies and applications are being developed. These technologies and applications are useful for data analysis and storage (Kalaian, 2019). Now, big data has become an interesting problem for researchers. Researchers are trying to define and represent big data in different ways (Mikalef, 2018).

The global big data market for software and services is expected to grow from $\$ 42$ billion to $\$ 103$ billion by 2027 . Every day 2.5 terabytes of data are generated, and in just the last two years, $90 \%$ of the world's data has been generated. And if that's any indication, there may be more to come (Getsmarter, 2019). 


\section{Big Data Market Woridwide Segment Revenue Forecast 2011-2026 \\ Big Data Market Forecast Worldwide from 2011 to 2026, by segment (in billion U.S. dollars)}

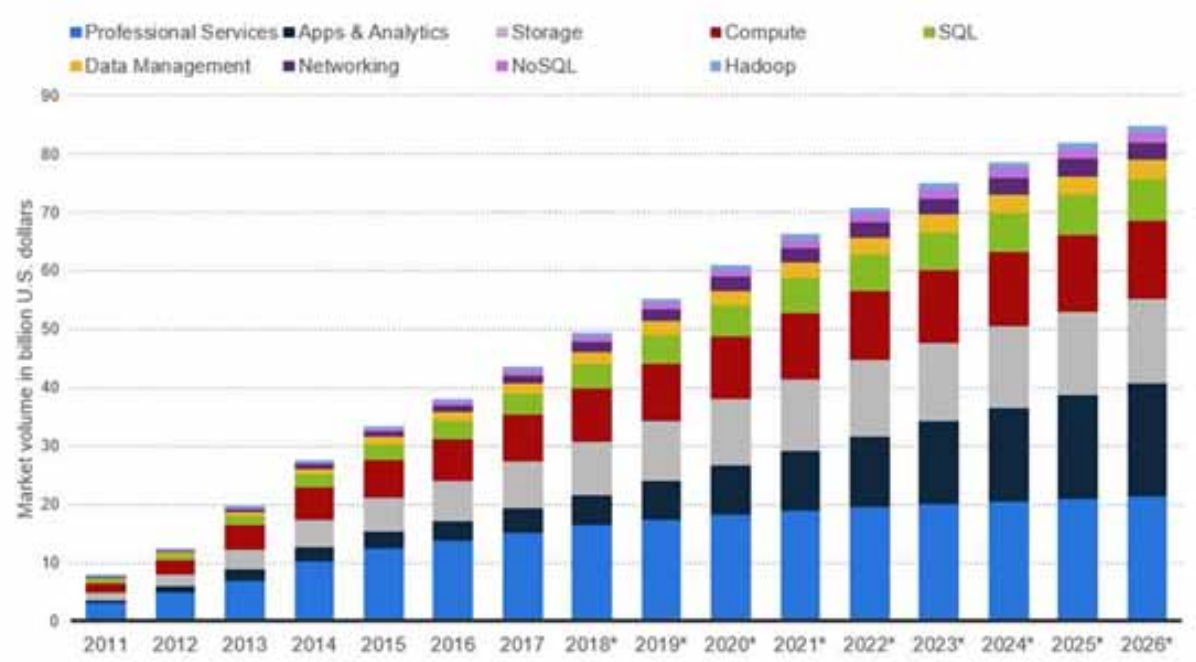

\section{statista5}

In education, huge amounts of data are generated through online courses, teaching and learning activities. With the advent of big data, teachers can now access students' academic performance, learning patterns and provide instant feedback (Black, 2018). Timely and constructive feedback motivates and satisfies students, which has a positive impact on their performance. Academic data can help teachers analyze their teaching methods and influence change according to the needs and demands of students. Many online education websites have been designed, and various courses have been launched according to students' personal preferences. Progress in the education sector depends on acquisitions and technology. Large-scale administrative data can play a huge role in managing all kinds of educational issues (Sorensen, 2018). Therefore, professionals must understand the effectiveness of big data in education to minimize educational problems.

There are many big data business and research platforms for students. However, most of these platforms do not serve students only, do not integrate students into school life, and do not teach students big ideas about AI, such as how computers perceive the world, represent knowledge, reason, and learn the world (Gardner-McCune, 2018).

China's AI industry is mainly divided into three layers: basic layer, technology layer and application layer. The application layer covers 20 fields such as enterprise technology integration and solution, smart business and retail, intelligent robot and intelligent hardware. In 2020, China's application-level AI enterprises accounted for $84.05 \%$ of the total, taking a leading position in the AI industry and guiding the development of the AI industry. (SHUAI, 2021)

The demand for big data is increasing significantly in different areas such as insurance and construction, healthcare, telecommunications and e-commerce. According to Dresner Consulting Services (Services, 2017), technology (14\%), financial services (10\%), consulting (9\%), healthcare 
(9\%), education (8\%) and telecommunications (7\%) were the most active industries in the process of generating large amounts of data. However, the educational sector is not an exception in this situation. In the educational realm, a large volume of data is produced through online courses, teaching and learning activities (Oi, 2017).

The big data and AI industries have not yet been combined much in China. The market leaders in big data and ai are both aggressively developing their fields. But the vast majority of resources are in the hands of the biggest companies, leaving the big data and AI industries locked in monopolistic competition. But the vast majority of the competition is in healthcare, drug discovery, business intelligence, gaming and manufacturing. In education, it's more about training and coaching, or big data analysis products developed for students. This enables our company to use the existing big data analysis technology combined with artificial intelligence to create products for the school environment and students' life. Our product is to program big data analyzed behaviour into artificial intelligence products, improving school teaching has become a major niche of our business.

$\mathrm{Al}$ and big data have the potential to enrich the field of education and training practice in a new way. In extreme cases, algorithms and intelligent machines replace (some) human educational tasks, which can mean that somewhat ironically, these algorithms and machines themselves have "received" some kind of education and training (for example, in the form of real-world data that helps them learn). On the other hand, educators may want to leverage AI and big data to enhance their educational practices in classrooms and other settings. Of course, this could also mean teaching ai/big data technologies relevant to education to better prepare future teachers for this new era (e.g., critically evaluating and interpreting AI results). There may be technical and proprietary aspects, but there are also ethical, social and ecological issues. If AI and big data really can change the way teachers think and behave, the field of education and training should be at the forefront of these developments. This may also involve other structures in society that promote an entrepreneurial culture (Audretsch, 2007).

From the perspective of regional education development needs, big data has strong practical significance and will become a new engine to drive future education development. Big data is becoming a new way to modernize school governance. Management disorder, efficiency is not high, the lack of technical support and the use of modern technology. Big data can not only operate and maintain all kinds of personnel information, education funds, school conditions and service management data of the school, but also comprehensively integrate, analyze and analyze these data, to provide data support for the scientific management of the school, to further improve the school level and school efficiency. Quality is the foundation of education. Using big data technology, we can comprehensively, truly and dynamically record the whole process of education and teaching. Through data analysis and application, we can effectively change the traditional education and teaching mode and make precise teaching possible. That is, we can help students more accurately understand themselves and develop themselves, from "fuzzy development" to "knowing well". Help teachers understand students more accurately, improve teaching, from "empiricism" to "data".

\subsection{METHODOLOGY}

We take the main data collection of quantitative research as our main research method. Quantitative research involves the empirical study of observable and measurable variables (JosephNg PS, 20122021). It is used for theoretical testing, predicting results, and determining relationships between variables using statistical analysis. In this method, data is collected by the researcher. Participants were recruited for the study, informed consent was obtained, and quantitative data were obtained electronically or in person by the researchers. This approach allows the researcher to determine exactly which variables he or she is interested in exploring and how to manipulate them in the study. Variables were measured using tools that have been established by other authors for psychometric characteristics (reliability and validity). Statistical techniques are used to analyze data to assess the nature of relationships between variables. 
Table 1. The five competitive forces model

\begin{tabular}{|c|c|}
\hline Competitive Forces & Explanation \\
\hline Bargaining Power of Suppliers & $\begin{array}{l}\text { Big data and artificial intelligence could be like a Swiss Army knife, } \\
\text { reducing the need for clunky tools. New suppliers may see demand for } \\
\text { specialized components of the Swiss Army Knife, but also include digital } \\
\text { services such as data services and analytics. As ai products increase and } \\
\text { data sets in big data grow, the bargaining power of new suppliers can be } \\
\text { high. But suppliers focus more on the future needs of their partners and the } \\
\text { company's capabilities, and only large manufacturers with deep pockets } \\
\text { can lead negotiations with suppliers. However, this disadvantage is offset } \\
\text { by factors such as low product differentiation among suppliers and the } \\
\text { competitive business environment suppliers face. }\end{array}$ \\
\hline Bargaining Power of Buyers & $\begin{array}{l}\text { Big data products create a direct link between companies and their } \\
\text { customers, reducing reliance on intelligence agents and weakening } \\
\text { the power of buyers. Customization can achieve lock-in by increasing } \\
\text { conversion costs. In short, differentiation becomes easier because other } \\
\text { values are easier to offer customers than price. }\end{array}$ \\
\hline The threat of New Entrants & $\begin{array}{l}\text { With widespread awareness of AI, AI and big data are likely to attract } \\
\text { potential entrants. However, the coding of big data and the software } \\
\text { for ARTIFICIAL intelligence are both high fixed costs. Although new } \\
\text { entrants cannot produce on the same scale as incumbents, they can } \\
\text { compete by offering niche products and niche markets. But if other giants } \\
\text { already produce your business, customers may not choose a smaller } \\
\text { company. For fast-growing technology companies, they sacrifice profits } \\
\text { to build more open solutions that don't lock in consumers and interoperate } \\
\text { with different solutions on the market. The shift to profiting from sales and } \\
\text { services may be more appropriate for players who specialize in software } \\
\text { development and spend little on hardware production. }\end{array}$ \\
\hline Threat of Substitutes & $\begin{array}{l}\text { The ability to see huge improvements in connected products can be a } \\
\text { threat to connected products, with fewer features serving niche needs, } \\
\text { or with products currently offered in the market that are impossible to } \\
\text { connect. Services may create new business models where you can rent a } \\
\text { product and few products serve the market. }\end{array}$ \\
\hline Rivalry Among Existing Firms & $\begin{array}{l}\text { The impact on competition may depend on the cost structure of the } \\
\text { company, the ways to differentiate will increase, but this may increase } \\
\text { fixed costs, such as more expensive software development. Increased } \\
\text { market intelligence may reduce variable costs because it makes it easier } \\
\text { to predict when customers will need to buy new equipment. To combat } \\
\text { the difficulty for actors in estimating the value of the latest technology to } \\
\text { consumers, companies may add unnecessary features. Both factors can } \\
\text { come at the expense of a company's profitability. }\end{array}$ \\
\hline
\end{tabular}

\subsection{FINDING \& DISCUSSION}

Griffith AI\&BD is positioned to sell to schools. Advanced Learning solutions are designed to improve the learning process based on students' large data sets, and combine ai technology to develop ai-enabled electronic devices. Schools need to provide the best learning experience to survive in a competitive world. We hope that these AI-assisted facilities and environments will be able to meet the learning needs of individual educational programs and schools. We will develop two aspects, one facing the school. With more accurate data, more products will be designed for students and parents.

Griffith AI\&BD collects large data sets on students and programs them into educational AI products. And produce a series of artificial intelligence products suitable for students around students. For example, the intelligent blackboard in the school, our artificial intelligence blackboard can give help to the teacher when he is lecturers, and for him to analyze the part that students are most interested 
in this topic, the most understandable way. And when the student goes to the blackboard to answer the questions, analyze his path and see how he interprets the questions. For example, we can use the intelligent test paper in the exam, analyze students' right and wrong, find students' shortcomings, analyze students' judgment on each question, analyze students' interest in each course, and recommend suitable majors for them in the future, and prevent cheating.

Our return on investment is correlated with positive cash flow over the long term. As with any major investment, we need to understand the life cycle and duration of ai products. We use a cost strategy to maximize product returns. Initially, we didn't have our student data set, so we had to buy educational and training institutions' data sets, and then program them into ai products and sell them to schools. Through the school, artificial intelligence will continue to learn to generate new data sets and then further upgrade our system of product generation. Because of the constant innovation and development of new technologies, we have to consider some $r \& d$ costs. The technical cost is $¥ 10000$ and the material cost is $¥ 2000$. According to the school market and our profit, the price of our product is $¥ 30,000$, which is more competitive than other AI and big data systems in the current market. Enough for us to get good returns and develop new technologies.

In the promotion activities, we use the form of cooperation with education and training institutions. Get educational institutions to adopt our products and experiment with students. Then we meet with schools, offer them the product, promote it, and let them experiment with it for free. We will also focus on using websites and social media to promote our brand. Our online service makes it easy for customers to contact us and they can even place wholesale orders through our website. End customers will also learn about our brand through the Internet.

We will take the online shopping situation for initial product sales. Because with the progress of The Times, people gradually pay more attention to network goods than offline goods. When the product develops to a certain extent, we will open physical stores in major malls to increase customer experience.

We are a B2B and B2C company. All schools are our customers, and all students will be our customers in the future. It uses the Internet to sell goods and services in a global society. We have online distributors and an advertise-based business-to-consumer model that uses free content to drive visitors to the site. In turn, these visitors are exposed to digital or online advertising. We also have sites like Facebook that build online communities based on shared interests to help marketers and advertisers advertise their products directly to consumers. The site will target ads based on a user's demographic and location.

We will identify our target market and contact them by phone and email to inform them of our business and products. If schools are interested in selling our products, we will visit them, or they can come to our company to discuss further details. While promoting products to schools, sales staff will provide free samples for educators to try, and provide a list of features for customers to browse our products. Once they confirm the order, we will prepare the order form to accommodate the spot order. Customers can choose to pay by cash, credit card or online banking. If they wish to reorder in the future, we also provide telephone orders and online order services, and then the order will be shipped directly to them. We intend to communicate with customers regularly to get their sales feedback, ask if they are willing to order again, and promote our new products to them.

When we find new customers, we send them an email to let them know that we appreciate what they see. Assure them that you will always be there for them and point out any problems they may have with the purchase. Also, pay attention to existing customers. Let them know that after winning their business, we still miss them. Contact them consistently without disturbing them. Well-orchestrated emails and promotions for your former clients can pay off handsomely. Turning one-time buyers into repeat customers is an excellent return on investment, and turning repeat customers into brand communicators is even better. They tell their friends and family how great your product or service is. That means free promotions. 
While there is a heated debate among educators, psychologists and parents about how much screen time is appropriate for children, it's another emerging technology in the form of artificial intelligence and machine learning that is starting to transform educational tools and institutions and change how what might happen in the future looks like in education. Ai in US education is expected to grow $47.5 \%$ from 2017 to 2021, according to the US Education Sector's AI Market report. Although most experts agree that the key presence of teachers is irreplaceable, there will be many changes in teachers' work and educational best practices (Marr, 2021).

Ai has been used in education mainly in tools that help develop skills and test systems. As AI solutions for education continue to mature, the hope is that AI can help fill gaps in learning and teaching needs, allowing schools and teachers to do more than ever before. Ai can improve efficiency, personalize and simplify administrative tasks, giving teachers the time and freedom to provide understanding and adaptability -- uniquely human capabilities that machines struggle to solve. By harnessing the best attributes of machines and teachers, ai's vision in education is that they work together to bring the best results for students. Because today's students need to work in a future where $\mathrm{AI}$ is a reality, our educational institutions must expose students to and use this technology.

The link between AI and education spans three areas: AI learning (e.g., using AI tools in the classroom), learning AI (its technologies and skills) and preparing AI (e.g., making all citizens better aware of the potential impacts) for ai's impact on human life. The "Teaching AI in Schools" project is currently focusing on the connection between the latter two. The goal is to promote the mainstreaming of the human and technical aspects of $\mathrm{AI}$ into school student training programs. It began by piloting the capacity development of curriculum developers and lead trainers in selected national institutions to empower young people.

The project plans to take three actions:

Developing an AI skills framework for schools;

Develop and manage an online repository to host selected AI-related training resources, the AI National Curriculum and other key digital skills training courses;

Seminars to support the integration of AI training into national or institutional school curricula in selected countries.

\subsection{CHALLENGES AND RISKS}

The first challenge is to develop a comprehensive view of sustainable development public policy. The complexity of the technical requirements for progress in this area requires the harmonization of multiple factors and institutions. Public policy must build partnerships at the international and national levels to create an AI ecosystem that serves sustainable development.

The second challenge is to ensure inclusiveness and equity in Al education. With the development of $\mathrm{Al}$, the least developed countries are at risk of suffering from new technological, economic and social divides and must face major obstacles such as basic technological infrastructure to lay the groundwork for the implementation of the new strategy of using $\mathrm{Al}$ for learning.

A third challenge is to get ready for artificial intelligence driver education teachers, and artificial intelligence to understand education, although it must be a two-way road: teachers must learn the new digital skills, the use of artificial intelligence in teaching and meaningful way, developers have to learn how teachers work, artificial intelligence and create sustainable solutions in the real environment.

The fourth challenge is to develop quality and inclusiveness. Data system. If we are going to digitise education, the quality of the data should be our number one concern. Developing national capacities to improve data collection and systematization is critical. The development of artificial intelligence should be an opportunity to increase the importance of data in the management of education systems.

The fifth challenge is to make the study of Al significant in education. It is reasonable to expect, though, that ai education research will increase in the coming years. 
Table 2. Projected development costs: fixed and variable

\begin{tabular}{|l|l|l|l|}
\hline \multicolumn{1}{|c|}{ Variable Cost } & \multicolumn{1}{c|}{$¥$} & \multicolumn{1}{c|}{ Fixed Costs } & \multicolumn{1}{c|}{$¥$} \\
\hline $\begin{array}{l}\text { Purchases Artificial } \\
\text { intelligence materials }\end{array}$ & 15000 & Salaries & 8000 \\
\hline Third-party big data sets & 4000 & Drawings (own salary) & 12000 \\
\hline & & Rent & 5000 \\
\hline & & $\begin{array}{l}\text { Utilities (telephone, } \\
\text { electricity, water) }\end{array}$ & 3000 \\
\hline & & Insurance & 2000 \\
\hline & & Advertising \& Promotions & 1000 \\
\hline & & Maintenance \& Upkeep & 1000 \\
\hline & & Interest payable & 0 \\
\hline Total & & Other expenses & 1000 \\
\hline
\end{tabular}

The sixth challenge concerns ethics and transparency in data collection, use and dissemination. $\mathrm{Al}$ raises several ethical issues relating to access to educational systems, advice to individual students, centralization of personal data, accountability, impact on work, data privacy, and ownership of data-feeding algorithms. As a result, Al regulations will require a public discussion about ethics, accountability, transparency, and safety.

\subsection{PROJECTED DEVELOPMENT COSTS}

The main source of revenue will be big data systems and artificial intelligence products for schools. The Big Data system is based on the adoption of big data sets in the education and training industry to get data on students. Combine campus production with artificial intelligence machines used on campus. The price of the big data service fee is $¥ 10,000$ per month, and the cost is $¥ 4,000$. Artificial intelligence shows the product decision, the price range is $¥ 1000-20000$, its cost is $¥ 200-10000$. The contribution rate is $¥ 6000$ and $¥ 800-10000$ respectively. The marginal profit comes from the selling price minus the cost of the goods sold, which will help cover the fixed costs and make a profit.

Variable costs are costs that change according to sales volume, while fixed costs are costs that remain constant regardless of sales volume or production volume.

Operating leverage boils down to an analysis of fixed and variable costs. Operating leverage was highest among companies with a high ratio of fixed operating costs to variable operating costs. These companies use more fixed assets in their operations. Conversely, the companies with the lowest ratio of fixed operating costs to variable operating costs had the lowest operating leverage. In the case of the enterprise, the proportion of its fixed costs is higher than the proportion of its variable costs. As a result, its operating leverage is considered high. High operating leverage means it takes longer for companies to break even. Therefore, enterprises should reduce their operating leverage ratio by reducing fixed costs, to minimize their risks and increase their stability.

The start-up costs required to start the business are $¥ 150,000$, which includes third-party data, taxes and licenses, furniture, accessories and equipment. Further details will be provided in the financial forecasts section.

This section describes the details of the operation of the company, how it will operate, and the methods of producing products and providing services to customers. 
The first step is to audit the internal data to find out what data is accessible and where it can be found.

The next step is to identify external data that can help the company with data modelling and analysis. Factor analysis can then be used to make the data easier to understand.

The next stage is to implement machine learning to gain insight from the data that has been acquired. Through machine learning, advanced analytics and normative analysis and optimization can be used to inform both near - and long-term decisions, identify customer needs, and ensure that development and marketing start-up expenditures are optimized to achieve maximum ROI.

The final stage is to integrate AI-driven decisions throughout the company. With the implementation of advanced analytics, companies can move from relying on leadership decisions to data-driven decisions throughout the organization. Employees across the organizational level must trust $\mathrm{AI}$ recommendations and be empowered to make these data-driven decisions.

The company will be based in the technology industrial park in Shenzhen, China. This strategic layout provides convenience for the development of our company because more high-tech products are released by companies in the industrial park.

Proprioception can also be called the self-awareness of thought, or the self-awareness of thought, the idea, being able to sense its flow, being aware of its movement. With proprioception, human emotional intelligence (EI) will also develop, which will gradually change human historical memory and add new elements to this historical memory at the level of intuitive thinking. By analogy, we can develop a similar philosophy of proprioception for AI. So we have to cultivate this awareness in everyone -- human or ai; We must "change" or establish certain ways of thinking (creative, critical and conscious thinking); It is important to start this process with the "youngest" possible age of the agent (human or AI). These capacities, which must be developed gradually, will enable us to deal with the daily needs of others and help raise awareness. This transformation/analogy is shown implicitly.

\subsection{MACHINE BEHAVIOUR AND EDUCATION}

Before any type of learning environment can be endowed with any kind of intelligence, machine ethics and/or machine behaviour must be integrated into that learning environment to ensure that the cognitive, social, and emotional abilities of defined students are formalized or translated in some way into a scientific language, a language familiar to machines.

In addition, methods must be defined to assess whether such intelligent systems work properly in the long term since it is impossible to notice in real-time or eliminate the impact of their failures or abnormal operations on the moral development of individuals. Because these methods, as noted earlier, are not in the domain of computer scientists, roboticists, and engineers who create machines, but in the hands of experts in the field of behavioural science, the roles of evaluators and auditors must take over from those of teachers. Therefore, teachers must be able to acquire certain knowledge from the field of ai behavioural science to be qualified observers and evaluators of this intelligent learning environment.

Artificial intelligence (AI) research involves the development of systems that are more or less intelligent and capable of solving relatively difficult problems. These methods are often based on imitating human problem-solving methods. In addition to machine learning, the field of AI includes knowledge representation, natural language understanding, automatic reasoning and theorem proving, logic programming, qualitative modelling, expert systems, gameplay, heuristic problem solving, artificial senses, robotics, and cognitive modelling.

Through the use of learning techniques, systems can learn and improve in perception, language understanding, reasoning and theorem proving, heuristic problem solving, and gameplay. The realm of logic programming is also highly related to inductive logic programming, which aims to develop logic programs from examples of target relationships. Also in qualitative modelling, machine learning algorithms are used to generate descriptions of complex models from examples of target system 
Figure 3. Transformation development

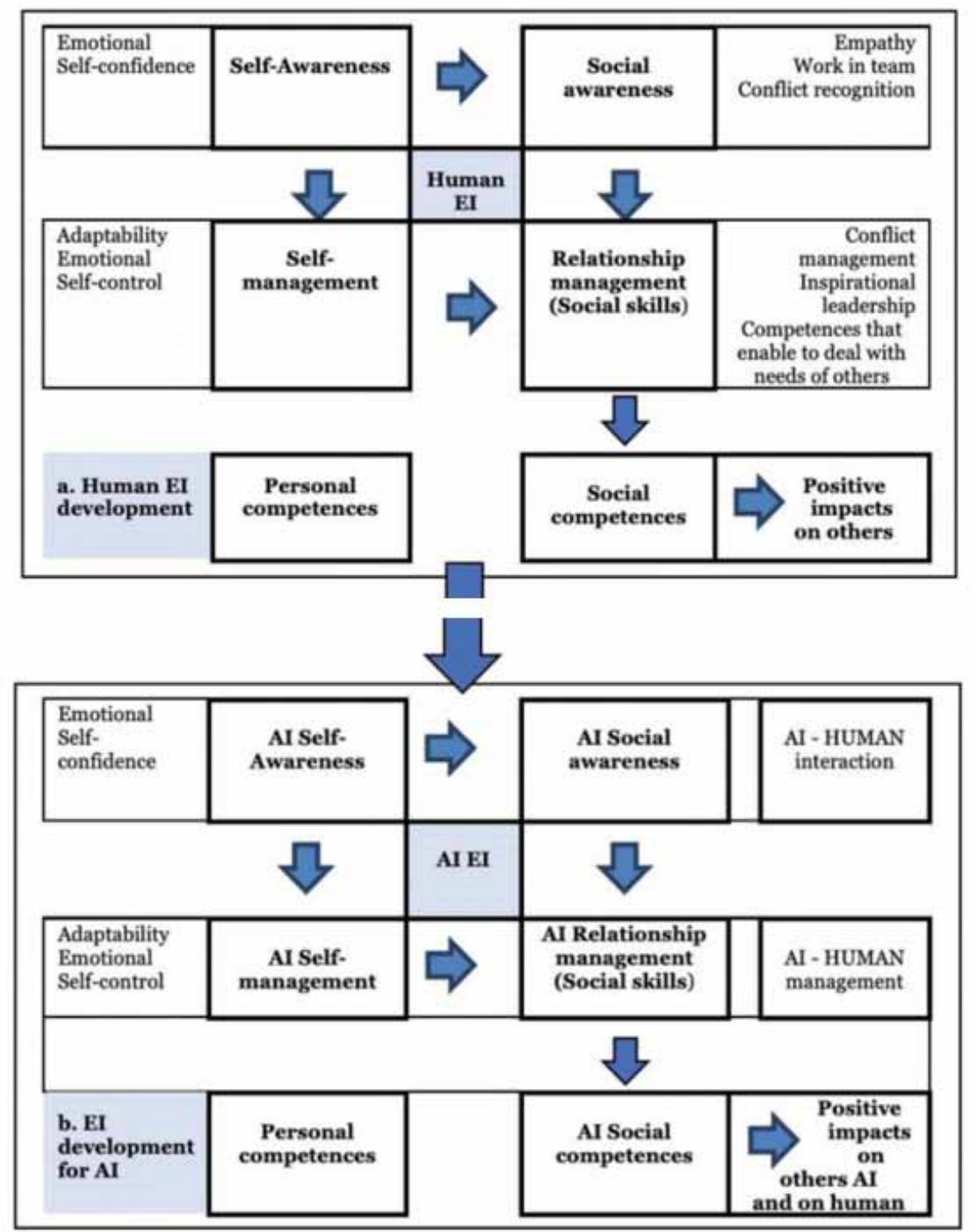

behaviour. For expert system development, machine learning can be used to generate a knowledge base from training examples of solved problems. Intelligent robots must inevitably learn to improve their problem-solving programs.

We developed ITS to consist of three permanent system modules (personalization, evaluation, and communication) and a module related to the topic at hand (topic module) that can be independently adjusted and/or changed by the teacher. The basic functions of each module are as follows:

The Personalization module (PM) is the connecting system between the individual learner and the learning system ITS. It is the link between teachers and learners, as well as between learners themselves during the participatory curriculum. The PM is closely related to the Evaluation module (EM). Its main task is to adapt learning path to individual learners (personalized and individualized), determine the initial state (on a particular subject (content) of knowledge level and the learner's attitude 


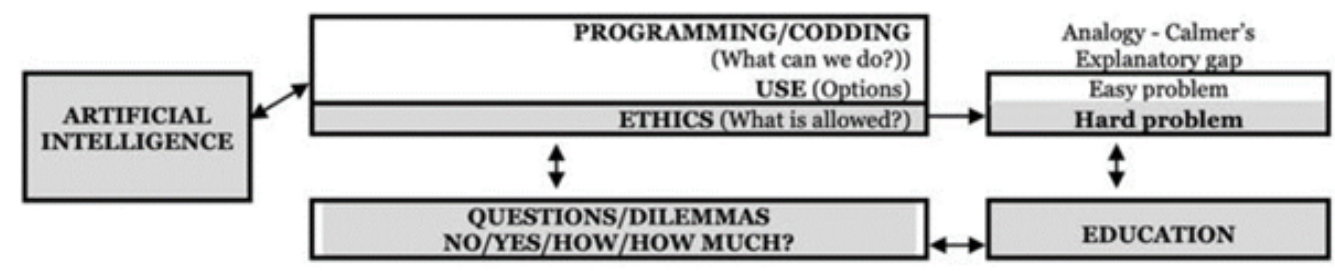

Figure 5. Structure of ES

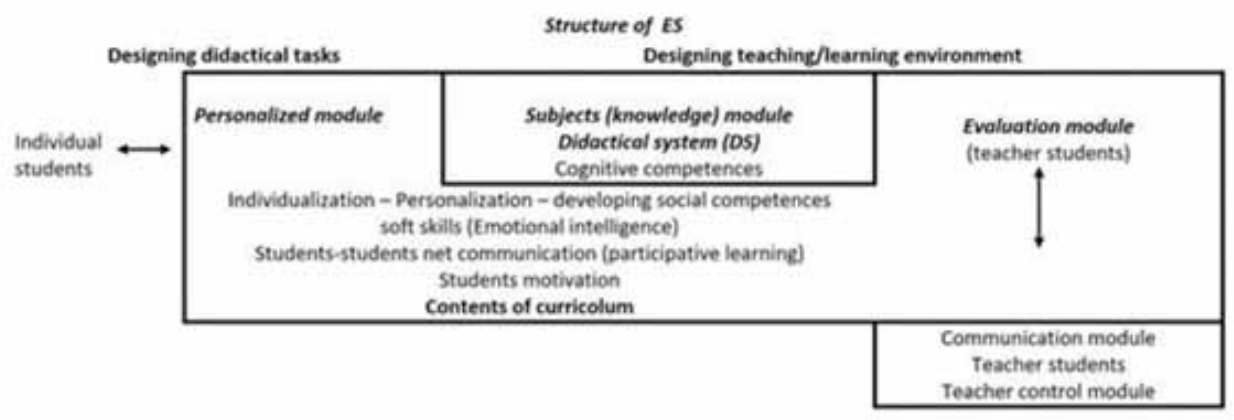

toward the subject), to monitor their progress and according to their needs (such as their learning style and ability to adjust learning path (differentiation). Since both system modules are based on artificial intelligence, the module is used to store an individual's data, and the learning system has been adapted from the beginning by modifying the subject module (SM) to fit his/her needs and abilities defined in the previous course (i.e., SM before). If all students had the same ITS at the beginning of the school year, we would have as many different ITS's at the end of the school year as the class. They will all achieve the same learning goals and reach the same learning standards, but they will take completely different paths to reach those goals.

\subsection{EVALUATION MODULE}

Analyze existing conditions

Student's knowledge (the cognitive component) and

Individual student decisions, awareness (social component, emotional intelligence).

And forward the results of these analyses to

Teachers who can monitor the progress of individual students (formative assessment),

Students, for self-assessment and motivation, and

The system, known as the Personalization Module (PM), is designed to personalize and personalize the learning path for individual students.

Subject modules (SM) are modules that are related to a particular topic, i.e. those that are related to specific teaching/learning content. The module consists of several elements (blocks) that are built on the idea of brain-based teaching/learning. Individual learning content (activities) is divided into 


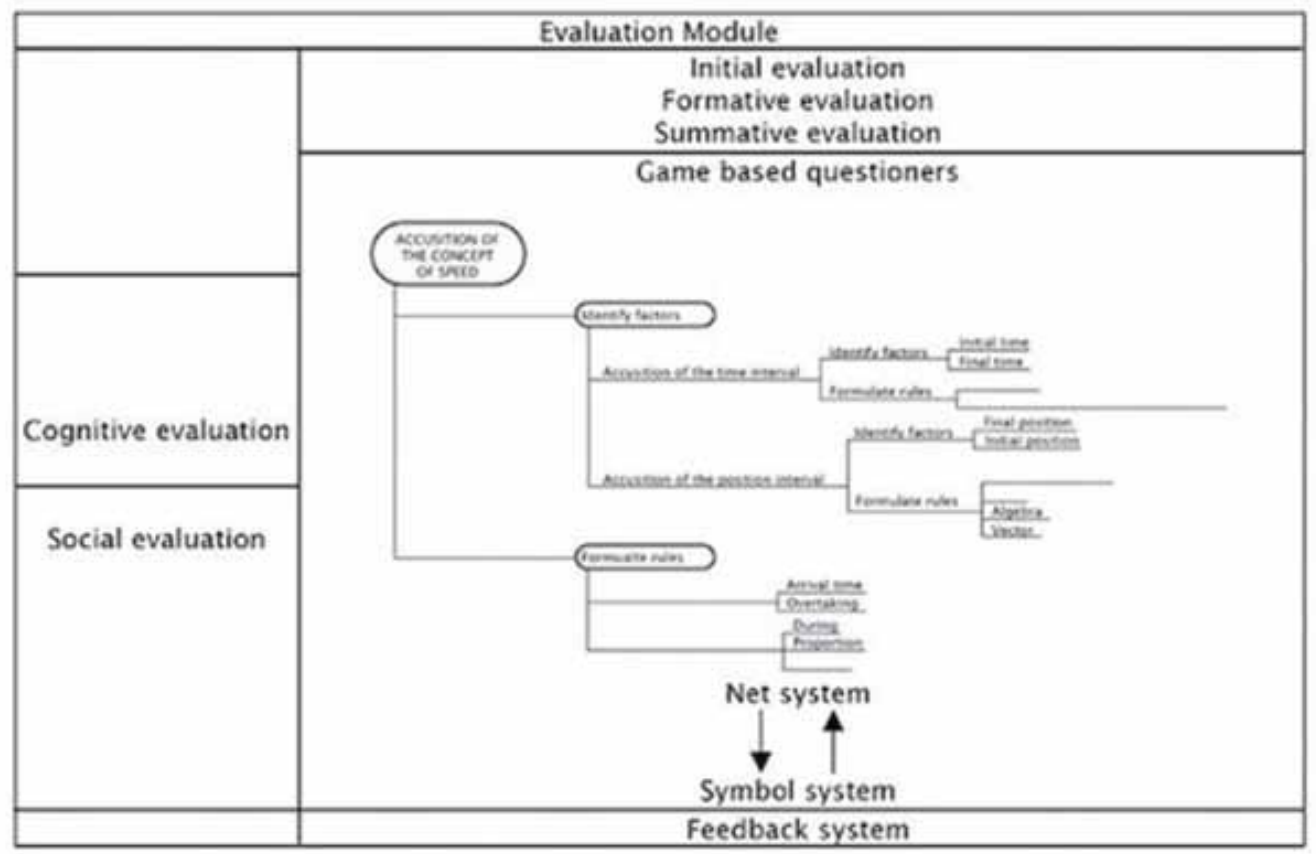

learning units of approximately 45 to 90 minutes, further including blocks of 10 to 20 minutes. Such modules can be organized for individual subjects as a whole (intelligent AI-textbook) or for individual issues or projects, stored in a database and available to teachers as teaching AIDS. These modules are set according to specific examples/learning situations to facilitate personal problems or research-based work (self-study, consolidating knowledge, assigning homework, etc.) for students engaged in formal (here, the module acts as a teaching aid to the teacher) or informal types of learning.

The basic structure of each block is shown in the figure below

\subsection{MANAGEMENT TEAM}

The management team of Griffith AI\&BD is composed of professional partners, professional technical personnel and investors.

\section{Founder, CEO and/or President}

The highest-ranking executive in the business,Some founders are also CEOs.

\section{Chief Operating Officer}

A high-level executive in a corporate community who oversees and manages daily activities.

\section{Vice President of Sales}

They oversee and direct the sales teams or departments to meet or exceed sales revenue. 
Figure 7. Subject module

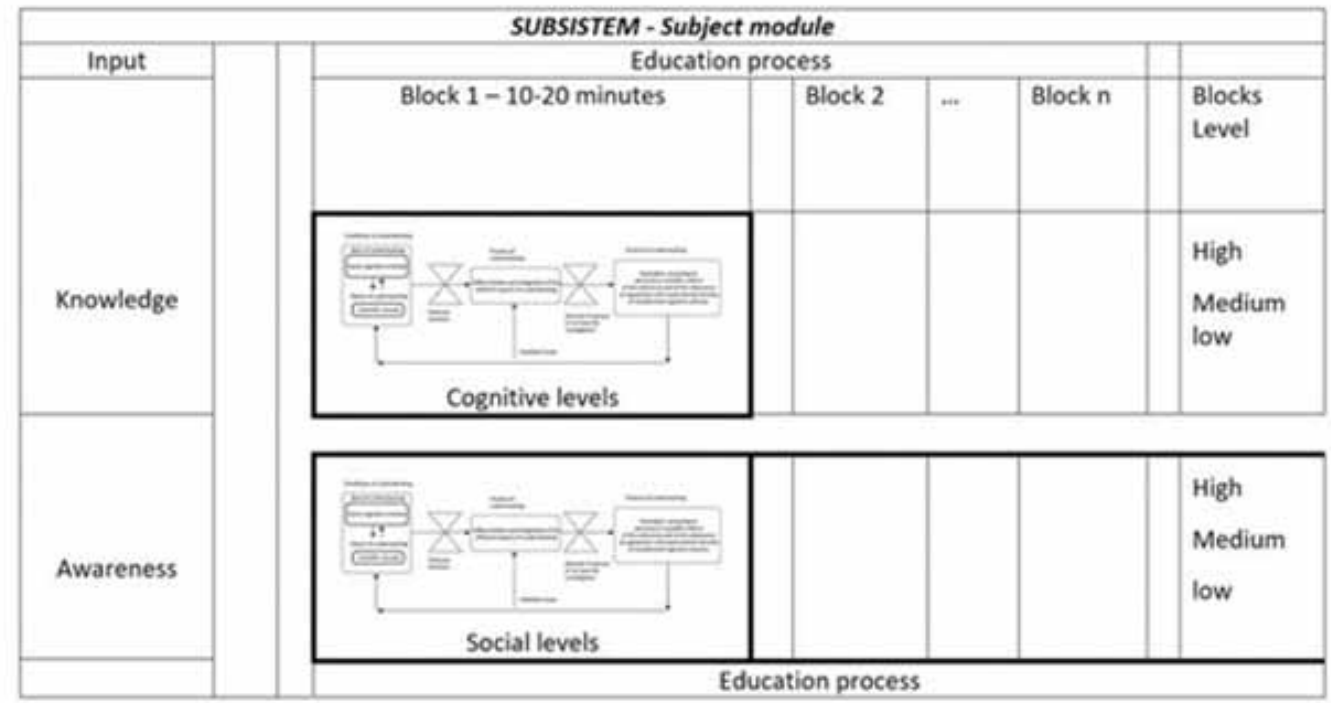

Figure 8. Subject module continued

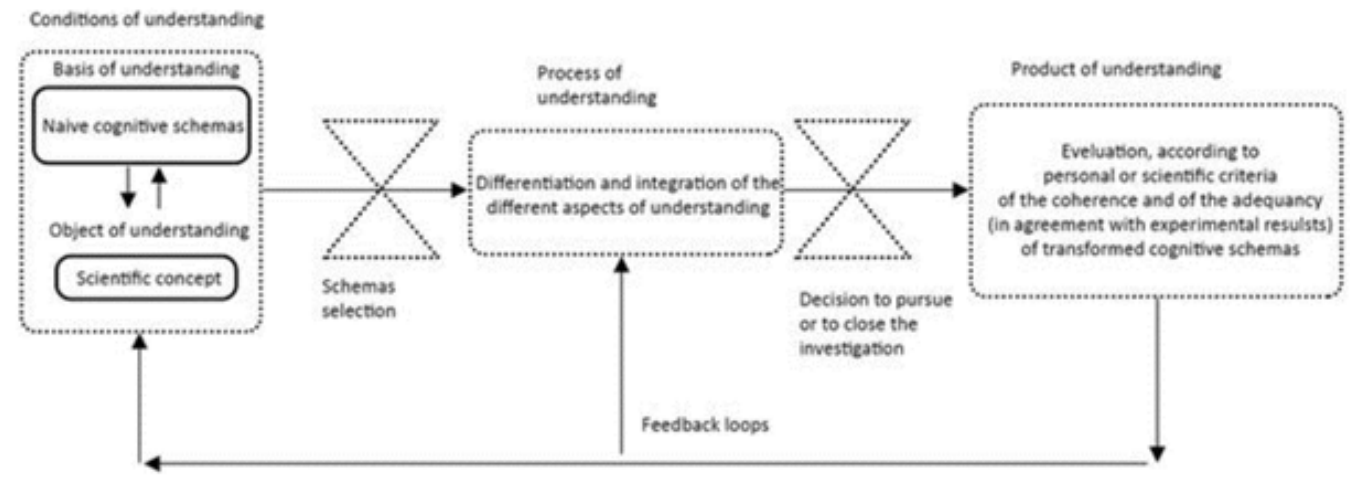

\section{Vice President of Marketing}

Oversee marketing strategies and efforts to strengthen the company's market position and achieve desired business objectives.

\section{Vice President of Web Development and/or Engineering}

Strong supervisory and leadership skills. Proficient with Microsoft Office Suite or related software. VPS of engineering is responsible for ensuring that design requirements are met, overseeing the consistency of user experiences, and managing a team of engineers and developers. 


\section{User Experience Designer/Manager}

User experience (UX) management is the practice of managing user experience design-related activities inside an organization to create growth and good management practices. Typical UX management activities are to define an organization's UX design language and strategy and manage the work processes around UX design.

\section{The product manager}

The Product Manager is responsible for the product planning and execution throughout the Product Lifecycle, including gathering and prioritizing product and customer requirements, defining the product vision, and working closely with engineering, sales, marketing and support to ensure revenue and customer satisfaction

\section{Digital Marketing Manager}

Digital marketing managers are responsible for implementing, overseeing, and managing digital marketing strategies that advance an organization's mission by reaching a broad audience and attaining greater exposure. The results of their efforts include increased sales, donations, or community interest and involvement.

\section{Human resource manager}

Human resource managers are the overseers of the human resources department and insurers of the functions and tasks being carried out by the HR team.

\section{Business Development Manager}

Business development managers are responsible for helping grow businesses by finding new opportunities, researching leads, establishing relationships with potential clients and arranging appointments for sales executives.

\section{Account Management/Customer Service Manager}

An account manager (AM) is a person who works for a company and is responsible for the management of sales and relationships with particular customers

\section{Sales manager/salesperson}

A sales manager is someone responsible for leading and guiding a team of salespeople in an organization. They set sales goals \& quotas, build a sales plan, analyze data, assign sales training and sales territories, mentor the members of his/her sales team and are involved in the hiring and firing process.

\subsection{ORGANIZATION STRUCTURE}

Startups have two of the most important roles -- CEO or founder and CTO. These two key players in the startup are at the top of our structure and have two different areas of responsibility. The CEO is responsible for:

Market sales

Legal stuff

The human resources

Integrated management

Monitor and communicate with CTO

In turn, the CTO oversees four mini-teams, namely:

User Experience team 


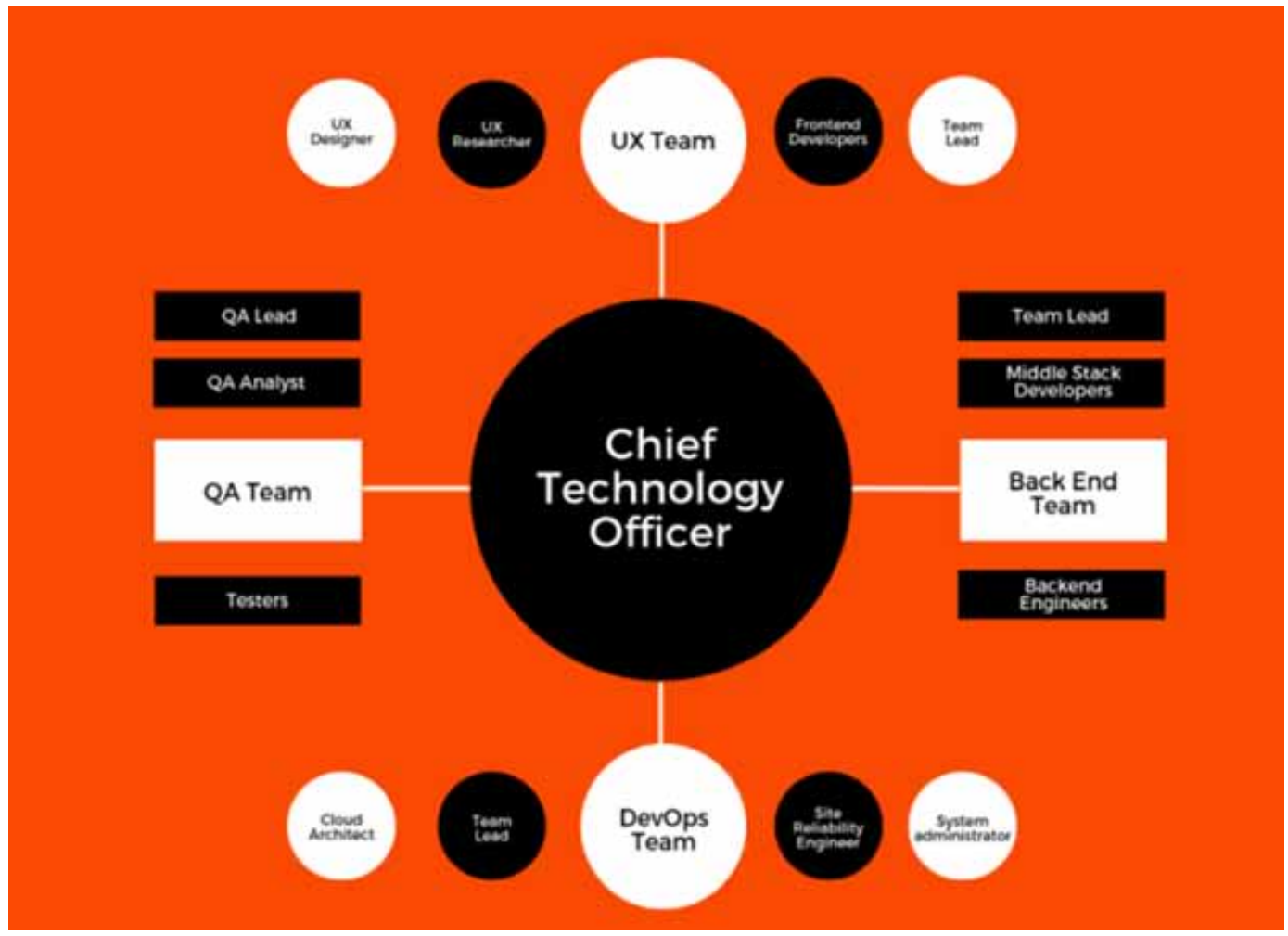

The backend team

Development and Operation team

Quality Assurance team

\section{User Experience Team}

User experience teams are an important part of almost every startup. It is responsible for creating the best experience for users through intelligent aesthetic design and front-end development. It also works with other mini-teams to achieve its goals. In our proposed model, the UX team includes the following startup team members:

User Experience Researcher

User Experience Designer

Front-end developer

The captain

\section{The Backend Teams}

Almost everyone in the tech world understands what a back-end team is and what it does. This team is one of the most important in the development department. It is responsible for the core of the application being built and the application logic that interacts with the cloud services and the back-end interacts with the front end through API Endpoints. Our proposed back-end team structure is as follows:

The group leader

Medium stack developer

Back-end engineer 
Table 3. Overall schedule-Gantt chart

\begin{tabular}{|c|c|c|c|c|c|c|c|c|c|c|c|c|c|c|c|c|c|c|}
\hline \multirow[t]{2}{*}{ Activities } & \multicolumn{5}{|c|}{ Jan. } & \multicolumn{4}{|c|}{ Feb. } & \multicolumn{5}{|c|}{ Mar. } & \multicolumn{4}{|c|}{ Apr. } \\
\hline & 1 & 2 & 3 & 4 & 5 & 1 & 2 & 3 & 4 & 1 & 2 & 3 & 4 & 5 & 1 & 2 & 3 & 4 \\
\hline $\begin{array}{l}\text { Conduct a feasibility } \\
\text { analysis }\end{array}$ & & & & & & & & & & & & & & & & & & \\
\hline Conduct a business plan & & & & & & & & & & & & & & & & & & \\
\hline Investment and Financing & & & & & & & & & & & & & & & & & & \\
\hline $\begin{array}{l}\text { Company business license } \\
\text { application }\end{array}$ & & & & & & & & & & & & & & & & & & \\
\hline Professional recruitment & & & & & & & & & & & & & & & & & & \\
\hline $\begin{array}{l}\text { Project establishment and } \\
\text { development }\end{array}$ & & & & & & & & & & & & & & & & & & \\
\hline $\begin{array}{l}\text { Launch the } \\
\text { business }\end{array}$ & & & & & & & & & & & & & & & & & & \\
\hline
\end{tabular}

\section{Development And Operation Team}

The primary responsibility of the DevOps team is cloud infrastructure management. They are responsible for adding new code to the repository and carefully testing it before deploying each new release. Speed to market and a perfect user experience are the main goals of the DevOps team. We propose the following DevOps team structure:

The group leader

Cloud architect

Field Reliability Engineer

System administrator

\section{Quality Assurance Team}

The quality assurance team is responsible for testing the application being built to ensure that there are no errors, errors, and stable and efficient performance. Therefore, it includes the following stakeholders:

The quality supervisor

Quality analyst

The tester

\subsection{OVERALL SCHEDULES}

\section{Gantt Chart}

Starting in January 2022

We will start a feasibility analysis in the first week of January 2022 to confirm the overall timetable of the business, which will take about 3 weeks. Then proceed with a three-week business plan. Investment and financing will take 4 weeks, and the company's business address lease and business license will be processed in two weeks. After the business license is completed, the recruitment of professionals will begin. Two weeks after the start of the recruitment of personnel, the project is established and started. In the third week of the project, commercial activities will start and the business will be fully developed. 


\subsection{FINANCIAL PROJECTIONS}

This section will show the company's financial plan.

Companies will invest in critical long-term equipment. It will be big data sets, big data updates, ARTIFICIAL intelligence product development, software development, system upgrades. According to the financial forecast, the start-up cost of Griffith AI\&BD business is $¥ 150,000$, and the monthly operating cost is $¥ 20,000$. The funding will be provided by partners.

Griffith AI\&BD’s financial reporting is based on these assumptions

A) The price of software and equipment has not changed significantly.

B) The business has operated in a period of sustained economic growth without a severe recession

C) The financial budget provided by the owner is sufficient to implement the financial plan.

D) No special policy requirements

According to financial forecasts, the business is expected to have 3.6 million in revenue in the first year and increase to 50,400,000 in the fifth year. Net profit is expected to increase from 178,600 in the first year to $25,256,320$ in the fifth year. This shows the profit of the business. Continuously increasing over time, operations increased from $4.96 \%$ in the first year to $50.11 \%$ in the fifth year.

Table 4. This section will introduce the startup cost, cash flow and profit of the company in 5 years

\begin{tabular}{|c|c|c|c|c|c|c|c|}
\hline \multicolumn{2}{|c|}{ Profit and loss Projections } & \multicolumn{2}{|c|}{ Year-1 } & Year-2 & Year3 & Year4 & Year5 \\
\hline \multicolumn{2}{|l|}{ Gross Revenue } & \multicolumn{2}{|c|}{$3,600,000$} & $7,200,000$ & $12,600,000$ & $25,200,000$ & $50,400,000$ \\
\hline \multicolumn{2}{|l|}{ Direct Cost $(10 \%)$} & \multicolumn{2}{|c|}{360,000} & 720,000 & $1,260,000$ & $2,520,000$ & $5,040,000$ \\
\hline \multicolumn{2}{|l|}{ Operating Income } & \multicolumn{2}{|c|}{$3,240,000$} & $6,480,000$ & $11,340,000$ & $22,680,000$ & $45,360,000$ \\
\hline \multicolumn{2}{|l|}{ Gross Margin } & \multicolumn{2}{|c|}{$90.00 \%$} & $90.00 \%$ & $90.00 \%$ & $90.00 \%$ & $90.00 \%$ \\
\hline \multicolumn{8}{|l|}{ EXPENSES } \\
\hline \multicolumn{2}{|l|}{ Payroll } & \multicolumn{2}{|c|}{500,000} & 600,000 & 700,000 & $1,000,000$ & $1,200,000$ \\
\hline \multicolumn{2}{|l|}{ R\&D Expenses } & \multicolumn{2}{|c|}{$1,200,000$} & $1,500,000$ & $2,500,000$ & $4,000,000$ & $6,000,000$ \\
\hline Marketing Expenses & & 500 & & 576,000 & 630,000 & $1,008,000$ & $1,512,000$ \\
\hline Professionals/Consultar & & 250 & & 300,000 & 350,000 & 400,000 & 500,000 \\
\hline Insurance Costs & & 30,0 & & 35,000 & 40,000 & 50,000 & 60,000 \\
\hline Management and Oper & 1 Cost & 300 & & 360,000 & 630,000 & $1,008,000$ & $1,008,000$ \\
\hline Depreciation/Amortiza & f Assets & 25,0 & & 35,000 & 50,000 & 70,000 & 90,000 \\
\hline Misc. Expenses & & 200 & & 250,000 & 300,000 & 500,000 & 750,000 \\
\hline Total Operating Cost & & 3,00 & 00 & $3,656,000$ & $\$ 5,200,000$ & $8,036,000$ & $12,128,000$ \\
\hline EBIT & & 235 & & $2,824,000$ & $6,140,000$ & $14,644,000$ & $33,232,000$ \\
\hline EBIT Margin (\%) & & 6,53 & & $39,22 \%$ & $48,73 \%$ & $58,11 \%$ & $65,94 \%$ \\
\hline Taxes $(24 \%)$ & & 56,4 & & 677,760 & $1,473,600$ & $3,514,560$ & $7,975,680$ \\
\hline Interest Expense & & 0 & & 0 & 0 & 0 & 0 \\
\hline Net Profit & & 178 & & $2,146,240$ & $4,666,400$ & $11,129,440$ & $25,256,320$ \\
\hline Ratio & Formul & & 1 & 2 & 3 & 4 & 5 \\
\hline Net Profit Margin (\%) & $\begin{array}{l}\text { Net prot } \\
\text { Revenu }\end{array}$ & ross & $4,96 \%$ & $29,81 \%$ & $37,03 \%$ & $44,16 \%$ & $50,11 \%$ \\
\hline
\end{tabular}




\section{REFERENCES}

Agrawal, J., \& Goldfarb, A.A. (2018). Prediction machines: The simple economics of artificial intelligence. Boston, MA: Harvard Business Press.

Audretsch, B. D. (2007). The entrepreneurial society. Oxford University Press. doi:10.1093/acprof:o so/9780195183504.001.0001

Bahirat. (2021). Top 10 Artificial Intelligence Trends in 2021. https://www.mygreatlearning.com/blog/topartificial-intelligence-trends/

Black. (2018). Classroom assessment and pedagogy. Assessment in Education: Principles, Policy \& Practice, 25(6), 551-575.

Casey. (2019). How big data and AI work together. https://enterprisersproject.com/article/2019/10/how-bigdata-and-ai-work-together

Chae, K. B. (2019). A general framework for studying the evolution of the digital innovation ecosystem: The case of big data. International Journal of Information Management, 45, 83-94.

Gardner-McCuneand \& Touretzky. (2018). Calypso for cozmo: Robotic Al for everyone (abstract only). Proceedings of the 49th ACM Technical Symposium on Computer Science Education, SIGCSE 2018.

Getsmarter. (2019). Big Data Analysis Techniques. https://www.getsmarter.com/blog/career-advice/big-dataanalysis-techniques/

Johnson. (2019). 5 Ways AI Is Changing The Education Industry. https://elearningindustry.com/ai-is-changingthe-education-industry-5-ways

Joseph, N. P. S., Choo, P. Y., Wong, S. W., Phan, K. Y., \& Lim, E. H. (2012). Malaysia SME ICT during economic turbulence. International Conference on Information \& Computer Network, 67-71.

Joseph, N. P. S., Mahmood, A. K., Choo, P. Y., Wong, S. W., Phan, K. Y., \& Lim, E. H. (2013). Battles in volatile information and communication technology landscape: The Malaysia small and medium enterprise case. International Journal of Business Information Systems, 13(2), 217-234.

Joseph, N. P. S., Mahmood, A. K., Choo, P. Y., Wong, S. W., Phan, K. Y., \& Lim, E. H. (2014). IaaS cloud optimization during economic turbulence for Malaysia small and medium enterprise. International Journal of Business Information Systems, 16(2), 196-208.

Joseph, N. P. S., Mahmood, A. K., Choo, P. Y., Wong, S. W., Phan, K. Y., \& Lim, E. H. (2015). Barebone cloud IaaS: Revitalization disruptive technology. International Journal of Business Information Systems, 18(1), 107-126. 
Joseph Ng, P. S., Choo, P. Y., Wong, S. W., Phan, K. Y., \& Lim, E. H. (2012). Hibernating ICT infrastructure during rainy days. J. Emerg. Trends Comput. Inf. Sci., 3(1), 112-116.

Kalaian, A., Kasim, R. M., \& Kasim, N. R. S. (2019). Descriptive and predictive analytical methods for big data. Web Services: Concepts, Methodologies, Tools, and Applications, 314-331.

Marr. (2021). How Is AI Used. In Education - Real World Examples Of Today And A Peek Into The Future. Bernard Marr \& Co.

Mikalef, I. O., Krogstie, J., \& Giannakos, MP. (2018). Big data analytics capabilities: A systematic literature review and research agenda. Information Systems and e-Business Management, 16(3), 547-578.

Ng. (2016a). Exostructure services for infrastructure resources optimization. J. Telecommun. Electron. Comput. Eng, 8(4), 65-69.

Ng. (2016b). Beyond barebone cloud infrastructure services: Stumbling competitiveness during economic turbulence. Journal of Science and Technology, 24(1), 101-121.

Ng. (2018). EaaS optimization: available yet hidden information technology infrastructure inside medium size enterprises. J. Technol. Forecast. Soc. Change, 132(July), 165-173.

Ng. (2019). EaaS infrastructure disruptor for MSE. International Journal of Business Information Systems, $30(3), 373-385$.

Ng. (2021). Making financial sense from EaaS for MSE during economic uncertainty. In K. Arai (Ed.), FICC 2021. AISC (Vol. 1363, pp. 976-989). Springer. https://doi.org/10.1007/978-3-030-73100-7_67.

Ng. (2021a). Economic turbulence \& EaaS grid computing. Int. J. Bus. Forecast. Market. Intell.

Ng. (2021b). Still technology acceptance model? Reborn: exostructure as a service model. International Journal of Business Information Systems.

$\mathrm{Ng}$, Loh, \& Eaw. (2020). Grid computing for MSE during volatile economy. International Conference on Control, Automation and Systems, 709-714.

Ng. (2016). EaaS: Available yet hidden infrastructure inside MSE. 5th International Conference on Network, Communication and Computing, ACM International Conference Proceeding Series, 17-20.

Ng, Kang, Choo, Wong, Phan, \& Lim. (2015). Beyond cloud infrastructure services in medium size manufacturing. International Symposium on Mathematical Sciences \& Computing Research, 150-155.

Ng-Poh-Soon, J., Yin, C. P., Wan, W. S., \& Nazmudeen, M. S. H. (2011). Energizing ICT infrastructure Malaysia SME during economic turbulence. Student Conference on Research and Development, 328-322.

Oi, M., Okubo, F., Shimada, A., \& Ogata, H.M. (2017). Reproducibility of findings from educational big data. Proceedings of the Seventh International Learning Analytics \& Knowledge Conference, 536-537.

Services Advisory Dresner. (2017). Big data adoption: State of the market. https://www.zoomdata.com/masterclass/state-market/big-data-adoption

Shuailiu. (2021). China's AI industry market development status in 2021 analysis of application needs to guide the development of ai industry. https://www.qianzhan.com/analyst/detail/220/210705-cff9fb10.html

Sorensen, C. L. (2018). "Big data" in educational administration: An application for predicting school dropout risk. Educational Administration Quarterly, 45(1), 1-93.

Wang. (2019). Competence for students' future: Curriculum change and policy redesign in China. ECNU Review of Education, 2, 234-245. 
Joseph Ng Poh Soon graduated with a Doctor of Philosophy (IT), Masters in Information Technology (Aus), Masters in Business Administration (Aus), and as an Associate Charted Secretary (ICSA-UK). He also holds various professional certifications that includes CCNP(SW), CCNA(RS), CCNA(Sec), CCAI, HCDA, HCNA, RSE, RCPS, VMCA, CEI, CEH, 3CE, MCP professional certification and is a member of MAICSA, ACM and IAENG. With his blended technocrat mix of both business knowledge and technical skills, he has held senior management position in many MNC and leads numerous mission critical systems. A humbly five times teaching excellence awards recipient with numerous research grants have leaded towards hundred plus publication citations. He has appeared in LIVE television prime time CyberSecurity talk show (RTM Bicara Politikonomi) and also had overseas teaching experience. His current researches are on strategic IT infrastructure optimization during the turbulence economy and digital transformations. 